\title{
Factores de Riesgo Biomecánicos y Psicosociales Presentes en la Industria Venezolana de la Carne
}

\section{BIOMECHANICAL AND PSYCHOSOCIAL RISK FACTORS IN THE VENEZUELAN MEAT PROCESSING INDUSTRY}

\author{
Mervyn Márquez Gómez'1, Miguel Márquez Robledo² \\ 1-2. Universidad Nacional Experimental del Táchira (UNET), San Cristóbal, Venezuela.
}

\begin{abstract}
RESUMEN
Introducción: la identificación y evaluación de factores de riesgo relacionados a trastornos musculoesqueléticos (TME) representa la base para una efectiva adaptación de las condiciones de trabajo. Objetivo: evaluar los principales factores de riesgo biomecánico y psicosocial a los que se exponen trabajadores de la industria cárnica. Método: el estudio es descriptivo, transversal, basado en observación directa, encuestas y análisis de videos, para la aplicación de las metodologías RULA, OCRA, ecuación de NIOSH, ERG0 y CoPsoQ-ISTAS21, sobre 71 tareas. Resultados: el mayor riesgo postural recae en tareas de embutido y empaque, alcanzándose puntajes RULA de 7. En cuanto a repetitividad destaca el empaque de productos cocidos con $60 \%$ de sus tareas en nivel medio. Los mayores riesgos por levantamiento de cargas se ubicaron en las áreas de embutido, con índices inaceptables entre 25\% y 50\% de sus tareas. En empujes y arrastres de cargas, cinco de las seis áreas presentaron niveles inaceptables de riesgo. Los factores psicosociales de inseguridad, doble presencia, estima y exigencias psicológicas fueron percibidos negativamente por más del 50\% de los trabajadores. Discusión: entre las principales causantes de los altos niveles de riesgo destacan: levantamiento de productos por encima de hombros, realización de actividades manuales de pre-empaque y el empuje manual de contenedores pesados. Conclusiones: el sector analizado reúne niveles de riesgo biomecánico y psicosocial que lo hace vulnerable a la aparición de TME.
\end{abstract}

(Márquez-Gómez M, Márquez-Robledo M, 2015. Factores de Riesgo Biomecánicos y Psicosociales Presentes en la Industria Venezolana de la Carne. Cienc Trab. Sep-Dic; 17 [54]: 171-176).

Palabras claves: FACTORES DE RIESGO; EVALUACIÓN; INDUSTRIA DE LA CARNE; INGENIERÍA HUMANA; SALUD LABORAL.

\section{ABSTRACT}

Introduction: the identification and assessment of risk factors related to Musculoskeletal Disorders (MSD) is the basis for an effective adaptation of working conditions. Objective: the aim is to evaluate the main biomechanical and psychosocial risk factors in workers of a meat processing industry. Method: the study is descriptive, transversal, based on direct observation, surveys and analysis of videos, for the application of the methodologies RULA, OCRA, NIOSH's equation, ERGO and CoPsoQ-ISTAS21, about 71 tasks. Results: the greater postural risk is in task of inlay and packaging, which reach RULA's scores of 7. In terms of repeatability, the packaging of products cooked stand out with $60 \%$ of its tasks at moderate level. The greatest risks by lifting were located in the inlay areas, with unacceptable rates between $25 \%$ and 50\% of its tasks. Five of the six areas showed unacceptable levels of risk in push and drag. The psychosocial factors: insecurity, double presence, esteem and psychological demands were perceived negatively by more than 50\% of the workers. Discussion: the main causes of the high levels of risk include: lifting of products above shoulders, manual activities of pre-packaging and the manual pushing of heavy containers. Conclusions: the meat industry has biomechanical and psychosocial risk levels that make it vulnerable to MSD.

Keywords: RISK FACTORS; EVALUATION; MEAT INDUSTRY; HUMAN ENGINEERING; OCCUPATIONAL HEALTH

\section{INTRODUCCIÓN}

Uno de los principales objetivos de los estudios epidemiológicos es la identificación de los factores que se asocian con el desarrollo o recurrencia de determinadas condiciones médicas, tal como las enfermedades musculoesqueléticas ${ }^{1}$; por lo tanto, la identificación

Correspondencia / Correspondence:

Conjunto Residencial Mi Refugio,

Torre 3, Apto 03Ph4, Via principal La Machiri, San Cristóbal, estado Táchira, Venezuela

Tel.: 58-416-6748510

e-mail: mervyn@unet.edu.ve; mervynmarquez@gmail.com

Recibido: 29 de septiembre de 2015 / Aceptado: 04 de noviembre de 2015

y evaluación de los factores de riesgo constituyen la base para lograr una efectiva adaptación de los puestos y condiciones de trabajo y, por ende, la prevención de muchos trastornos de esta naturaleza. ${ }^{2}$

Se pueden identificar tres grupos principales de factores que pueden aumentar el riesgo de trastornos musculoesqueléticos, estos son los factores biomecánicos o físicos, los factores organizativos y psicosociales, y los factores individuales o personales ${ }^{3,4}$; dentro de los factores biomecánicos pueden mencionarse la aplicación de fuerza, los movimientos repetitivos, las posturas forzadas y estáticas, y otros vinculados a condiciones del entorno de trabajo; en el grupo de los factores organizativos y psicosociales figuran el nivel de exigencia, la falta de control sobre el trabajo, el nivel de satisfacción y la falta de apoyo de supervisores y compañeros; mientras que los factores individuales guardan relación con el historial médico del trabajador, su edad, obesidad, tabaquismo, entre otros. 
Aun cuando la etiología multifactorial de los trastornos musculoesqueléticos ha sido aceptada, los factores de tipo biomecánico siguen representando el centro de atención como los principales responsables de la aparición de este tipo de enfermedad ocupacional. Los factores de riesgo físico más importantes a los que se encuentran expuestos los trabajadores son las posturas forzadas, los movimientos repetitivos, la manipulación de cargas y la realización de fuerzas importantes. ${ }^{5}$

De esta forma, cualquier contexto de trabajo en que se evidencie uno o varios de estos factores constituirán trabajos con gran potencial para desarrollar o agravar trastornos musculoesqueléticos en sus trabajadores. Aunque en general, la industria manufacturera constituye la actividad económica más afectada en Venezuela con 53,5\% de los casos $^{6}$, el sector alimenticio y en particular el dedicado a la producción y procesamiento de productos cárnicos presenta características especiales que lo hacen aun más susceptible, como son: altos volúmenes de producción, ciclos de trabajo relativamente cortos, tareas con alto contenido manual, alta manipulación manual de productos en proceso y productos terminados pesados $\mathrm{y}$, en algunos casos, procesos artesanales.

El objetivo planteado en este estudio es evaluar los principales factores de riesgo biomecánicos y psicosociales a los que se encuentran expuestos los trabajadores del área operativa de una empresa representativa de la industria cárnica venezolana, específicamente dedicada al embutido de productos. Dentro de los factores biomecánicos se incluyen la sobrecarga postural, la repetitividad de movimientos y la manipulación de cargas.

\section{MÉTODO}

La investigación desarrollada tiene un nivel descriptivo, ya que se enfocó en la valoración de los principales factores de riesgo identificados en los distintos puestos de trabajo analizados, permitiendo caracterizar así la situación actual de la empresa. En cuanto al diseño de investigación, referido al plan concebido para obtener la información, se empleó un diseño no experimental, dado que no se manipularon las variables sino que se describieron los elementos en su contexto natural. Adicionalmente, la investigación tuvo un diseño transeccional o transversal, ya que los datos fueron recopilados en un momento determinado. ${ }^{7}$

La población objeto de estudio la conforma los 145 trabajadores pertenecientes a las áreas de producción, que se encontraban laborando en la empresa al momento del estudio; se excluyeron a los trabajadores del área administrativa, por no ser del interés de la investigación. La muestra estuvo conformada por 67 trabajadores, seleccionados de acuerdo a un muestreo aleatorio estratificado por área de trabajo (error del 10\% y confiabilidad de 95\%), según la distribución señalada en la Tabla 1. Las áreas evaluadas son seis:

Tabla 1.

Distribución de trabajadores y tareas.

\begin{tabular}{lcc|} 
Área & $\mathrm{N}^{\circ}$ Trabajadores & $\mathrm{N}^{\circ}$ Tareas evaluadas \\
\hline Almacén MPNC & 4 & 2 \\
\hline Embutido cocido & 15 & 16 \\
Embutido crudo & 12 & 11 \\
Jamones & 6 & 11 \\
Empaque cocido & 14 & 10 \\
Empaque crudo & 16 & 21 \\
Total & $\mathbf{6 7}$ & $\mathbf{7 1}$
\end{tabular}

almacén de materia prima no cárnica (MPNC), embutido de productos cocidos, embutido de productos crudos, embutido de jamones, empaque de productos cocidos y empaque de productos crudos.

Para la recolección de los datos se emplearon distintas técnicas tales como la observación directa, la encuesta y la filmación de tareas, como base para la aplicación de los métodos de evaluación ergonómica utilizados; todo ello bajo los protocolos de investigación establecidos en la Declaración de Helsinki. Al respecto, se seleccionó un método de evaluación para cada tipo de factor: la sobrecarga postural se evaluó mediante el método RULA, la repetividad de movimientos a través del método Check List OCRA, el levantamiento de cargas utilizando la ecuación revisada de $\mathrm{NIOSH}$, el empuje y arrastre de cargas con el método ERGO IBV y, finalmente, los factores psicosociales de acuerdo a la versión corta del ISTAS21 (CoPsoQ). A cada nivel de riesgo obtenido a partir de la aplicación de los métodos le fue asociado un color referencial específico, desde verde, que indica un nivel de riesgo leve, hasta rojo, que simboliza un nivel de riesgo alto.

\section{Método RULA (Rapid Upper Limb Assessment)}

EL método RULA se basa fundamentalmente en mediciones angulares realizadas sobre las posturas más significativas adoptadas por el trabajador durante el desarrollo de sus tareas; para ello debe observarse y seleccionarse aquellas posturas que a priori supongan una mayor carga postural. El método divide el cuerpo humano en dos grupos: el grupo A que incluye miembros superiores (brazos, antebrazos y muñecas) y el grupo B que abarca las piernas, el tronco y el cuello. A cada grupo se asigna una puntuación global, la cual puede modificarse por el tipo de actividad muscular desarrollada y de la fuerza aplicada. ${ }^{8}$

La puntuación final resultará en un número comprendido entre $1 \mathrm{y}$ 7 , proporcional al riesgo que implica la realización de la tarea, donde los valores altos indican riesgos mayores de aparición de lesiones musculoesqueléticas. A partir de este puntaje, el método establece niveles de actuación comprendidos entre 1 y 4: el nivel 1 indica una postura aceptable; el nivel 2 que pueden requerirse cambios en la tarea; el nivel 3 que se requiere el rediseño de la tarea, mientras que el nivel 4 indica que se requieren cambios urgentes.

\section{Método Check List OCRA (Occupational Repetitive Action)} El método Check List OCRA es una evaluación rápida que permite determinar el riesgo asociado a la repetitividad de movimientos, con énfasis en los miembros superiores del cuerpo. Este método basa su veredicto en función del análisis de los siguientes factores: duración real del movimiento repetitivo, periodos de recuperación, frecuencia de las acciones, duración y tipo de fuerza ejercida, postura de miembros superiores, así como la consideración de otros factores adicionales como vibraciones, precisión, $\mathrm{y}$ ritmo de trabajo. ${ }^{9}$

El resultado final del método es un valor numérico denominado indice check list OCRA, a partir del cual se asocia un nivel de riesgo diferente: óptimo, aceptable, muy ligero, ligero, medio y alto, de manera que mientras mayor sea índice, mayor es el riesgo asociado.

\section{Ecuación revisada de NIOSH (National Institute for Occupational Safety and Health)}

La ecuación de NIOSH permite evaluar tareas en las que se realicen levantamientos de cargas. La ecuación establece que en condiciones ideales de levantamiento el peso máximo recomen- 
dado es de $23 \mathrm{Kg}$; por lo que en la medida en que las características del levantamiento se alejen del ideal, el peso disminuirá. Los factores considerados incluyen el peso de la carga, las distancias horizontal y vertical existentes entre el punto de agarre de la carga y la proyección sobre el suelo, el ángulo de asimetría, la frecuencia de levantamientos, la duración del levantamiento y los tiempos de recuperación, así como el tipo de agarre.

El método ofrece como resultado el peso máximo recomendado que puede levantarse bajo las condiciones dadas, para evitar la aparición de trastornos musculoesqueléticos a nivel de la espalda. A partir de este valor se obtiene además el índice de levantamiento, que permitirá valorar el riesgo que implica la tarea para el trabajador. ${ }^{10}$

\section{Método ERGO IBV}

El método ERGO IBV (Instituto de Biomecánica de Valencia) para la evaluación de la manipulación manual de cargas, específicamente relacionado a tareas de empuje y arrastre, se basa a su vez en: la guía técnica para la evaluación y prevención de los riesgos relativos a la manipulación de cargas del Instituto Nacional de Seguridad e Higiene en el Trabajo, las tablas de pesos y fuerzas máximos aceptables de Snook y Ciriello, y la norma UNE-EN 1005-2. Las variables contempladas por el método incluyen la fuerza inicial, la fuerza sostenida, la distancia recorrida, la frecuencia de los empujes o arrastres y la altura de agarre de la carga. ${ }^{11}$

El método permite encontrar un índice que representa el nivel de riesgo asociado a la tarea de empuje o arrastre, según sea el caso, clasificándolo en aceptable, moderado o inaceptable.

\section{Método CoPsoQ-ISTAS21}

Para el caso de los factores psicosociales se empleó la versión corta del cuestionario de evaluación de riesgos psicosociales en el trabajo CoPsoQ-ISTAS21 (Instituto Sindical de Trabajo, Ambiente y Salud), a través de la aplicación informática ERGO IBV. ${ }^{12}$ Este método considera seis dimensiones psicosociales: exigencias psicológicas, trabajo activo y posibilidades de desarrollo, inseguridad, apoyo social y calidad de liderazgo, doble presencia y estima.

Los niveles de exposición psicosocial vienen dados por las puntuaciones obtenidas en cada dimensión, cuyos puntajes y rangos varian de una dimensión a otra, a partir de las cuales puede obtenerse tres niveles: más favorable, intermedio y más desfavorable.

\section{RESULTADOS}

La identificación inicial de los factores de riesgo se llevó a cabo mediante la observación directa de las principales tareas del área operativa de la empresa, encontrándose en general factores de riesgo relativos a sobrecarga postural, repetitividad de movimientos, levantamiento de cargas pesadas, y empuje y arrastre de cargas pesadas. En el caso de los factores psicosociales, debido a la dificultad de determinar por observación directa si los mismos pudieran representar un factor de riesgo determinante, se consideró como un factor a ser evaluado en todas las tareas.

\section{Sobrecarga postural}

Con relación al factor de sobrecarga postural, es importante acotar que en cada tarea se consideraron y evaluaron mediante el método RULA las posturas adoptadas por el trabajador, conside-
Figura 1.

Distribución del riesgo postural por área de trabajo.

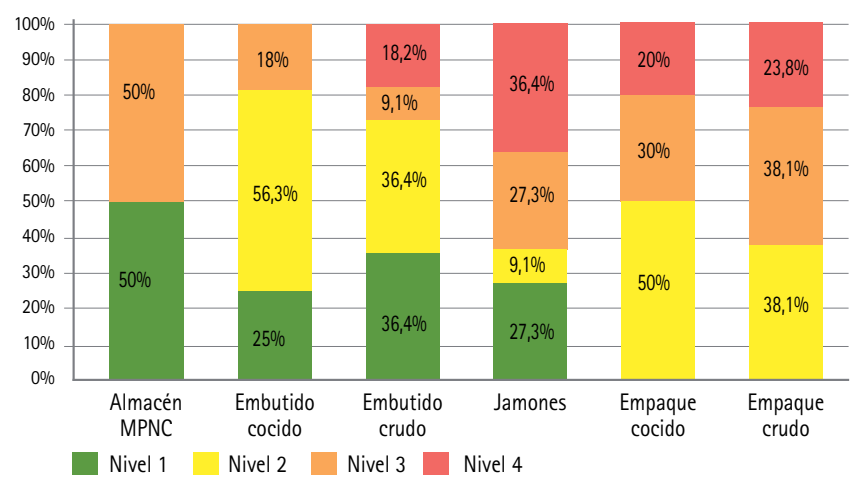

radas a simple vista como más fatigantes, y que podían referirse al lado derecho del cuerpo, al lado izquierdo o ambos; sin embargo, el nivel de actuación asignado a la tarea corresponde al puntaje más alto obtenido para alguna postura específica. En la Figura 1 se pueden observar los resultados de las evaluaciones de sobrecarga postural (71 tareas) de acuerdo a cada área de trabajo. Puede apreciarse que las áreas de mayor riesgo desde el punto de vista de sobrecarga postural son embutido de jamones y las áreas de empaque, tanto de productos cocidos como crudos, encontrándose que en cada una de ellas al menos el 50\% de sus tareas se ubican en un nivel de actuación de 3 o 4 . Al revisar los niveles de riesgo por tipo de tarea (ver Figura 2) resaltan, en primer lugar, las tareas de inyección, perteneciente al área de jamones (100\% de las posturas analizadas se ubican en nivel 4), seguida de las tareas de embalaje que abarca el encajado y paletizado de productos (50\% en nivel 4) y luego las tareas relacionadas a la operación de las líneas de embutido (33,3\% en nivel 4).

Al analizar las características comunes de estos grupos de posturas, se constata que predomina el levantamiento de productos por encima de los hombros para su ubicación en los carros transportadores o en la máquina de inyección, lo que implica la extensión de los brazos por encima de $90^{\circ} \mathrm{y}$ extensión del cuello, además de cargas manejadas comprendidas entre 2 y $10 \mathrm{Kg}$.

\section{Repetitividad de movimientos}

La evaluación concerniente a la repetitividad de movimientos se efectuó sobre las 71 tareas en estudio mediante el método Check List OCRA, a partir del cual se clasificó cada tarea en función del indice obtenido, en seis posibles niveles de riesgo. En la Figura 3

Figura 2.

Distribución del riesgo postural por tipo de tarea.

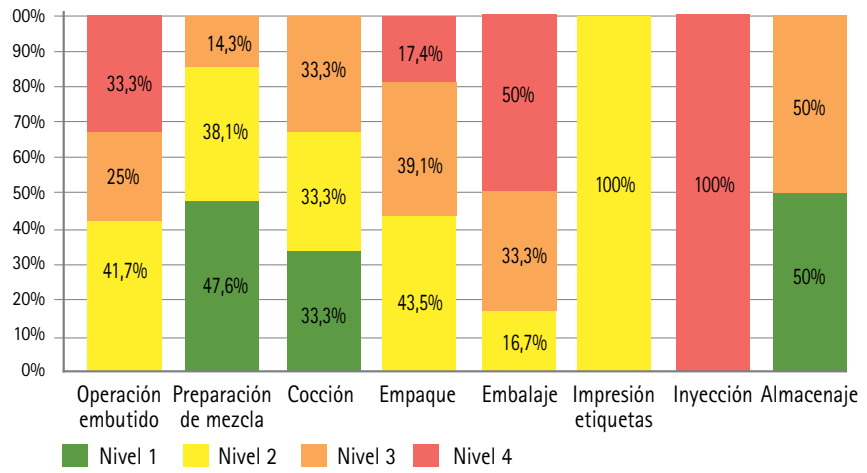


Figura 3.

Distribución del riesgo de repetitividad por área de trabajo.

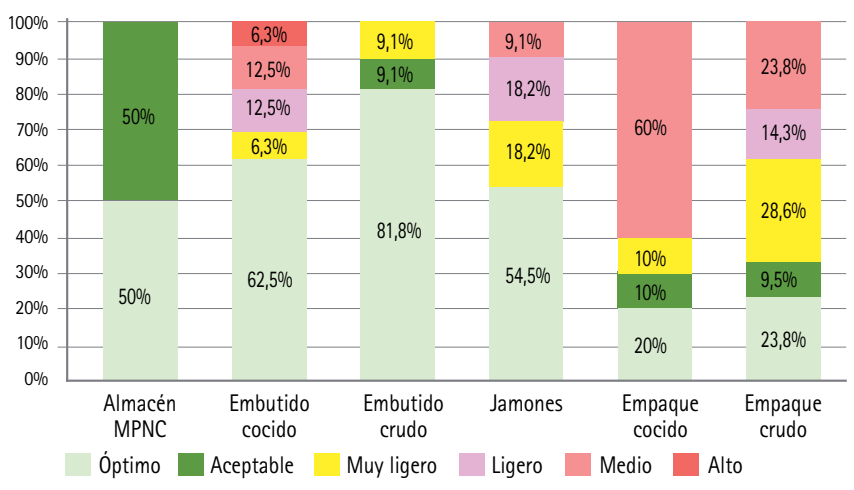

se presenta el resumen de las evaluaciones realizadas por área de trabajo, donde se puede observar que el área de empaque de productos cocidos aglomera la mayor proporción de tareas (60\%) con riesgo asociado a movimientos repetitivos. Una característica particular de esta área es la necesidad de realizar un conjunto de actividades de preparación del producto antes de su empaque definitivo, que incluyen el corte y retirado manual del pabilo que une las piezas, la separación de cada unidad y su colocación en estuches; lo que amerita que los dedos de la mano realicen un agarre tipo pinza casi todo el tiempo, además de la ausencia de pausas adecuadas de recuperación.

Otras áreas con riesgo medio o alto en repetitividad son el empaque de productos crudos (23,8\%), el embutido de productos cocidos $(18,8 \%)$ y el embutido de jamones $(9,1 \%)$. Puede evidenciarse diferencias entre los niveles de riesgo por repetitividad en el embutido de productos cocidos y de jamones, respecto al embutido de productos crudos (que no presentó riesgo), lo cual puede explicarse por el nivel de mecanización de las tareas; el embutido de productos cocidos se realiza de una forma más artesanal.

\section{Levantamiento de cargas}

El factor de riesgo referido al levantamiento manual de cargas se analizó a través de la ecuación de NIOSH en un total de 30 tareas que ameritan realizar un esfuerzo de esta naturaleza. Las áreas de empaque resultaron ser las más favorables al presentar la mayor proporción de tareas con un índice de levantamiento aceptable (ver Figura 4); mientras que las áreas de embutido de crudos, cocidos y jamones mostraron los valores más desfavorables (niveles de riesgo inaceptable y moderado).

Figura 4.

Distribución del riesgo de levantamiento de cargas por tipo de tarea.

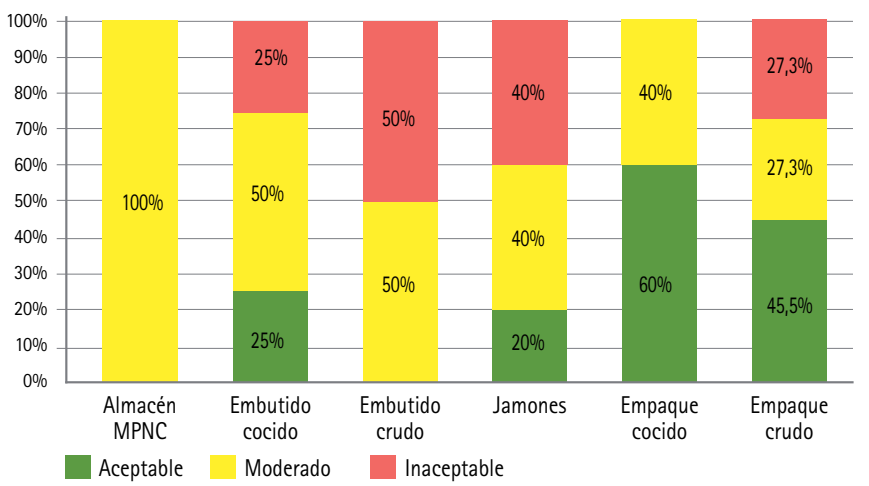

$\mathrm{Al}$ analizar las causas de estos resultados se encontró que, al igual que en la evaluación de posturas, las actividades relacionadas con la carga y descarga de productos en los carros transportadores registran los índices de mayor riesgo. Estos carros transportes son estructuras metálicas rodantes de hasta 5 niveles, en los cuales se ubican los diferentes tipos de productos (jamones, salchichas, chorizos, chuletas), dispuestos sobre travesaños metálicos, y que permiten su movilización prácticamente a lo largo de la planta de producción, e inclusive para actividades de horneado y almacenaje. Los factores de la ecuación de NIOSH que se ven afectados primordialmente son el factor de desplazamiento vertical, ya que el nivel superior del carro transportador puede llegar a los $185 \mathrm{~cm}$ de altura; además del correspondiente factor de desplazamiento horizontal y el peso de las cargas.

\section{Empuje y arrastres de cargas}

La evaluación del factor de riesgo relacionado a los empujes o arrastres de cargas se realizó sobre un total de 28 tareas, en las que se identificó este factor, mediante el método ERGO IBV. Los resultados presentados en la Figura 5 muestran que, a excepción del área de empaque de productos cocidos, en todas las áreas se presentó por lo menos una tarea con un nivel de riesgo inaceptable en empujes o arrastres de cargas. Resalta que en el almacén de materia prima no cárnica la única tarea analizada presentó este nivel de riesgo, seguido por el área de embutido de cocido (3 tareas) y jamones (2 tareas).

Figura 5.

Distribución del riesgo de empujes/arrastres de cargas por tipo de tarea.

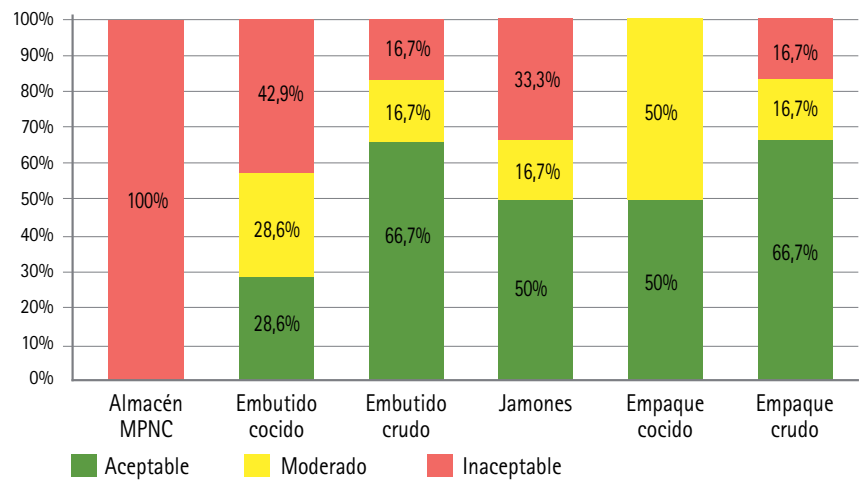

Como es de esperarse, las causas de estos resultados están directamente vinculadas con las fuerzas de empuje o arrastre, iniciales y sostenidas, requeridas para movilizar las cargas (fuerzas iniciales de hasta $64 \mathrm{Kg}$ y sostenidas de hasta $34 \mathrm{Kg}$ ). Se pudo comprobar que, además de las fuerzas, otro denominador común en los empujes o arrastres en nivel inaceptable es el tipo de carga movilizada; por lo general, se trata de contenedores metálicos rectangulares de $100 \mathrm{~cm}$ de lado y $110 \mathrm{~cm}$ de altura, los cuales son movilizados con transpaleta manual, generalmente sobre pisos grasos y húmedos, en los que se transporta materia prima utilizada en las fases iniciales del proceso.

\section{Factores psicosociales}

A diferencia de los factores de riesgo analizados anteriormente, los factores psicosociales fueron evaluados por los trabajadores de las distintas áreas de la empresa, y no hacen referencia a una tarea en particular propiamente dicha. En total fueron consultados un 
Figura 6.

Distribución de riesgos psicosociales por dimensión.

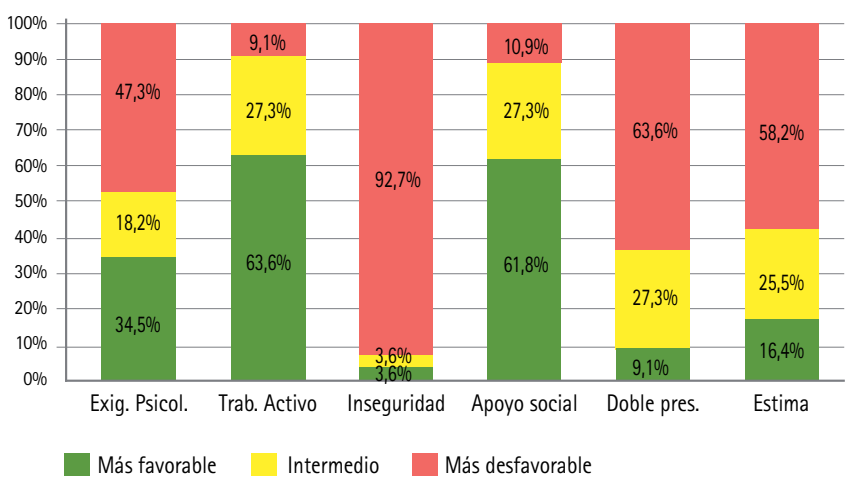

conjunto de 55 trabajadores, haciendo uso del cuestionario del ISTAS21, cuyos resultados se presentan en la Figura 6.

En general, puede apreciarse que las dimensiones que resultaron más desfavorables son la de inseguridad, doble presencia y estima. La inseguridad, referida no sólo a la inseguridad contractual sino a la inseguridad relacionada con las condiciones de trabajo, fue valorada como desfavorable por el 92,7\% de los trabajadores. Por su parte, la doble presencia, relacionada con la percepción del trabajador de una doble carga de trabajo (el trabajo remunerado en la empresa y las exigencias derivadas del ámbito familiar y doméstico), fue valorada como desfavorable por el 63,6\% de los trabajadores. Mientras que la estima, referida al respeto y reconocimiento de los superiores por el esfuerzo realizado, fue evaluada como desfavorable por el 58,2\% de los encuestados.

El trabajo activo y posibilidades de desarrollo, y el apoyo social y calidad de liderazgo fueron las dimensiones percibidas por los trabajadores encuestados como más favorables. La primera, referida a la influencia sobre el trabajo, control de tiempo, posibilidades de desarrollo, sentido del trabajo e integración en la empresa, fue percibida como favorable por el 63,6\% de los trabajadores; la segunda, que involucra a variables tales como la claridad de rol, previsibilidad, apoyo social, posibilidades de relación social, sentimiento de grupo y calidad de liderazgo fue valorada como favorable por el $61,8 \%$ de los sujetos.

\section{DISCUSIÓN}

Los resultados hallados en el estudio permiten confirmar que el sector de la industria cárnica, representado por la empresa analizada, presenta factores de riesgo biomecánicos y psicosociales que lo hacen vulnerable a la aparición de trastornos de tipo musculoesqueléticos en sus trabajadores, ya que se encontraron niveles de riesgo alto desde el punto de vista postural, de repetitividad y de manipulación de cargas, todos ellos asociados a una mayor prevalencia de desórdenes musculoesqueléticos ${ }^{5,13,14}$; unido a niveles desfavorables en algunos factores psicosociales tales como la inseguridad, la doble presencia, la estima y las exigencias psicológicas, igualmente vinculados a este tipo de enfermedad ocupacional. ${ }^{15,16}$

En lo práctico, la investigación realizada advierte sobre la necesidad de efectuar acciones de mejora, en algunos casos con carácter de urgencia, de forma tal que permitan reducir los niveles de riesgos a valores aceptables y por consecuencia reducir el riesgo de trastornos musculoesqueléticos. Las recomendaciones en cuanto al factor de riesgo postural deben orientarse principalmente al rediseño de facilidades de transporte que eviten la adopción de posturas fatigantes por encima de hombros. Por su parte, las recomendaciones relacionadas al riesgo por repetitividad de movimientos deben dirigirse fundamentalmente a la consideración de tiempos de recuperación mediante la inclusión de pausas en el trabajo. Entre tanto, las recomendaciones asociadas al levantamiento de cargas deben concentrarse en el análisis de los pesos de las cargas, así como en las distancias verticales, lo que nuevamente guarda estrecha relación con los dispositivos de transporte de productos usados actualmente.

Para mitigar los riesgos debido a empujes o arrastres de cargas es conveniente considerar la incorporación de equipos de transporte mecánicos tales como transpaletas eléctricas o montacargas, que permitan sustituir los esfuerzos humanos. Finalmente, con relación a los factores psicosociales deben considerarse algunas acciones tales como: proporcionar estabilidad en el empleo y evitar cambios inconsultos sobre las condiciones del trabajo, reconocer el trabajo realizado, flexibilizar las jornadas de trabajo en función de las necesidades particulares del trabajador y establecer procedimientos concretos orientados a un trato justo del personal. No obstante, este estudio pretende constituir una base para el desarrollo, análisis e implementación de estrategias dirigidas a empresas pertenecientes a este ramo industrial, por lo que los planes de intervención ergonómica deben realizarse en el interior de la misma empresa, de una forma participativa entre las distintas partes involucradas.

En lo que respecta a la continuidad de esta investigación, surgen nuevas inquietudes enfocadas a la prevención de trastornos musculoesqueléticos, a partir de los niveles de riesgo biomecánico y psicosocial presentes en un determinado trabajo. En otras palabras, deben desarrollarse modelos predictivos que incluyan la interacción de los elementos significativos, y permitan pronosticar si una persona será víctima o no de la enfermedad. ${ }^{17}$

\section{CONCLUSIONES}

Los mayores riesgos asociados a sobrecarga postural se encuentran vinculados a tareas que implican la carga o descarga de carros transportadores utilizados en la movilización de productos en proceso, en especial las posturas relacionadas al acceso de los niveles superiores e inferiores de los mismos; de igual forma se encontró un riesgo alto en tareas de paletizado de productos, debido a la ubicación de productos en los niveles inferiores.

Los mayores riesgos vinculados a la repetitividad de movimientos se hallaron en tareas relacionadas a la preparación de productos para su empaque y en ciertas tareas de las líneas de embutido, en especial de productos cocidos.

Los riesgos más altos relacionados al levantamiento de cargas se encuentran en tareas que implican la carga y descarga de productos en los carros transportadores; mientras que los riesgos más resaltantes debido a empujes o arrastres de cargas recaen en las movilizaciones de materias primas en tanques metálicos, realizadas con transpaleta manual.

Los factores psicosociales que resultaron más desfavorables en las áreas evaluadas fueron la inseguridad, la doble presencia y la estima, percibidas negativamente por más del 50\% de los trabajadores encuestados. 


\section{REFERENCIAS}

1. Piedrahita H. Evidencias epidemiológicas entre factores de riesgo en el trabajo y los desórdenes músculo-esqueléticos. Mapfre Medicina. 2004; 15:212-221.

2. Asensio S. Metodología para la generación de agendas de rotación de puestos de trabajo desde un enfoque ergonómico mediante algoritmos evolutivos [tesis]. Valencia, España: Universidad Politécnica de Valencia; 2009.

3. Agencia Europea para la Seguridad y la Salud en el Trabajo. Introducción a los trastornos musculoesqueléticos de origen laboral [en línea]. Bilbao: AESST; 2007. FACTS, 71 [consultado 5 feb 2015]. Disponible en: https://osha.europa.eu/ es/publications/factsheets/71.

4. Bell A, Steele J. Risk of musculoskeletal injury among cleaners during vacuuming. Ergonomics. 2012; 55(2):237-247.

5. Díez M, Garasa A, Macaya M, Eransus J. Trastornos músculo-esqueléticos de origen laboral. Pamplona: Instituto Navarro de Salud Laboral; 2007.

6. Instituto Nacional de Prevención, Salud y Seguridad Laborales. Registro enfermedades ocupacionales Año 2006. Caracas: INPSASEL; 2006 [consultado 4 ene 2015]. Disponible en: http://www.inpsasel.gob.ve/moo_medios/ sec_estadisticas.html.

7. Hernández R, Fernández C, Baptista P. Metodologia de la investigación. México: McGraw-Hill; 2010.

8. McAtamney L, Corlett N. RULA: a survey method for the investigation of workrelated upper limb disorders. Appl Ergon. 1993; 24(2):91-99.

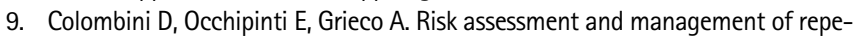
titive movements and exertions of upper limbs. Amsterdam: Elsevier; 2002.
10. Ergonautas.com [sitio web en Internet]. Valencia: Universidad Politécnica de Valencia; 2006. Niosh; Ecuación revisada de Niosh [consultado 20 ene 2015]. Disponible en: http://www.ergonautas.upv.es/metodos/niosh/niosh-ayuda.php.

11. Ergo/ IBV. Evaluación de riesgos ergonómicos. Valencia: Universidad Politécnica de Valencia; 2011.

12. Instituto Sindical de Trabajo, Ambiente y Salud. Manual del método CoPsoQistas21 (versión 1.5) para la evaluación y prevención de los riesgos psicosociales. Barcelona: Centro de Referencia de Organización del Trabajo y Salud ISTAS; 2010.

13. Attwood D, Deeb J, Danz M. Ergonomic solutions for the process industries. Burlington: Elsevier; 2004.

14. Zorrilla V. Trastornos musculoesqueléticos de origen laboral en actividades mecánicas del sector de la construcción [tesis]. Extremadura: Universidad de Extremadura; 2012.

15. Ahlgren $C$, Malmgren E, Brulin C. Gender analysis of musculoskeletal disorders and emotional exhaustion: interactive effects from physical and psychosocial work exposures and engagement in domestic work. Ergonomics. 2012; 55(2):212-228.

16. Rodríguez C, Gutiérrez L, Cortés M, Cerda E. Estudio de la carga global de trabajo y percepción de molestias músculo-esqueléticas en embarazadas atendidas en la unidad de maternidad del hospital. Cienc Trab. 2011; 13(39):53-56.

17. Keerthi N, Babski K. Effects of personal, psychosocial and occupational factors on low back pain severity in workers. Int J Ind Ergon. 2014; 44:335-341. 